\title{
Cumulative cost of prescription medication in outpatients with type 1 diabetes in Finland
}

\author{
R. Lithovius • V. Harjutsalo • C. Forsblom • \\ P. H. Groop • on behalf of the FinnDiane Study Group
}

Received: 18 June 2010 / Accepted: 8 November 2010/Published online: 7 December 2010

(C) Springer-Verlag 2010

\begin{abstract}
Aims/hypothesis We examined the 11-year cumulative outpatient cost of prescription medication in patients with type 1 diabetes by subgroups according to the presence of complications and duration of diabetes.

Methods This longitudinal study included a nationally representative cohort of patients with type 1 diabetes $(N=3,717)$ from the Finnish Diabetic Nephropathy Study (FinnDiane). The data were linked to the Drug Prescription Register. The cumulative cost was calculated between 1998 and 2008. Information on complications was updated until 2008. Patients were divided into 10-year groups according to the duration of diabetes in 1998. Generalised linear mixed
\end{abstract}

Electronic supplementary material (ESM) The online version of this article (doi:10.1007/s00125-010-1999-y) contains a list of physicians and nurses at each of the FinnDiane centres participating in patient recruitment and characterisation, which is available to authorised users.

R. Lithovius • V. Harjutsalo • C. Forsblom • P. H. Groop ( $\triangle)$ Folkhälsan Institute of Genetics, Folkhälsan Research Center, Biomedicum Helsinki, Helsinki University,

Haartmaninkatu 8 (C318b),

PL 6300014 Helsinki, Finland

e-mail: per-henrik.groop@helsinki.fi

R. Lithovius · V. Harjutsalo • C. Forsblom • P. H. Groop Department of Medicine, Division of Nephrology,

Helsinki University Central Hospital,

Helsinki, Finland

V. Harjutsalo

Diabetes Prevention Unit,

National Institute of Health and Welfare,

Helsinki, Finland

P. H. Groop

The Baker IDI Heart and Diabetes Institute,

Melbourne, Australia models under gamma distribution were used to evaluate the costs.

Results Approximately $25 \%$ of the patients had macrovascular disease (MVD) and/or end-stage renal disease (ESRD). The adjusted cumulative cost of medications increased 56\% when MVD was present compared with those without complications. In patients with ESRD or with both complications present the cost increased fourfold or 15-fold, respectively, when diabetes medications were excluded. The proportion of renal failure related medications (immunosuppressants, peritoneal dialytics and erythropoietin) accounted for more than $70 \%$ of these costs. The cost of diabetes medication was rather stable, irrespective of complication status or duration of diabetes. However, when complications were present these costs were markedly lower in all 10-year duration groups.

Conclusions/interpretation This study shows that ESRD has a great impact on outpatient prescription medication costs. Since no considerable differences were observed in the cost of diabetes medication, the increase was completely due to the cost of medications related to comorbidity.

Keywords Comorbidity · Complications - Cumulative costs - Duration of diabetes · End-stage renal disease .

Macrovascular disease $\cdot$ Outpatient $\cdot$ Prescription medications · Type 1 diabetes

$\begin{array}{ll}\text { Abbreviations } \\ \text { ATC } & \text { Anatomic Therapeutic Chemical Classification } \\ & \text { System } \\ \text { DPR } & \text { Drug Prescription Register } \\ \text { eGFR } & \text { Estimated glomerular filtration rate } \\ \text { ESRD } & \text { End-stage renal disease } \\ \text { FinnDiane } & \text { Finnish Diabetic Nephropathy Study } \\ \text { GLMM } & \text { Generalised linear mixed model }\end{array}$




$\begin{array}{ll}\text { MDRD-4 } & \begin{array}{l}\text { The modification of diet in renal disease } \\ \text { equation }\end{array} \\ \text { MVD } & \text { Macrovascular disease }\end{array}$

\section{Introduction}

The incidence of type 1 diabetes is increasing on average by $2.5-3 \%$ per year worldwide $[1,2]$. If this alarming increase continues, the number of new cases diagnosed below the age of 15 years, in Finland as well as throughout Europe, will double within the next 15 years [3, 4]. Among Finnish children, the incidence rate of type 1 diabetes is the world's highest; at the beginning of the millennium the incidence exceeded 60/100,000 per year [3]. Hence, an increasing number of patients will have to cope with diabetes from childhood throughout their lifetime.

Approximately one-third of patients with type 1 diabetes develop diabetic nephropathy, which is frequently associated with retinopathy and neuropathy as well as cardiac and cerebrovascular diseases [5]. These long-term micro- and macrovascular complications are the major cause of premature mortality, morbidity, reduced quality of life and increased healthcare costs [6]. Furthermore, type 1 diabetes is also associated with other chronic conditions such as autoimmune diseases and psychiatric disorders [7]. Consequently, patients with type 1 diabetes are often prescribed a variety of other medications that individuals without diabetes do not use [8-11]. Prescription medication constitutes the second largest component of the direct cost of type 1 diabetes after the cost of hospitalisation [12,13]. The cost of prescription medication has been estimated to cover approximately one-third of the total cost of type 1 diabetes in Finland [12].

In order to be able to optimise the use of healthcare resources, policymakers should be familiar with the cost structure of diabetes care. Therefore, a systematic, longitudinal follow-up of cost is an essential tool for resource planning. Exploration of the changes in medication costs over time, and identification of the main elements that contribute to these costs, could help policymakers to focus their attention on the major cost drivers related to type 1 diabetes and its complications. This in turn could lead to a better utilisation of available healthcare resources and would allow the development of strategies to reduce the incidence and progression of diabetic complications and the associated costs.

Cross-sectional studies have shown higher utilisation and cost of prescription medications by patients with diabetes than by non-diabetic individuals $[8,10,11]$. In these register-based studies, where the majority of patients have had type 2 diabetes, the use and cost of medications have been compared between diabetic and non-diabetic individuals during a 1-year period. Longitudinal studies are, however, rare in the field of medication-cost analysis.

Obviously, patients with type 1 diabetes at different stages of disease severity and duration are likely to require different levels of resources. Thus, it is important to identify potential subgroups of patients with type 1 diabetes in order to improve our understanding of the cost structure of diabetes care. Using longitudinal data from a nationally representative cohort of adults with type 1 diabetes, we therefore estimated the 11-year cumulative outpatient cost of prescription medication according to patients' complication status and duration of diabetes.

\section{Methods}

Patients participated in the Finnish Diabetic Nephropathy Study (FinnDiane), an ongoing nationwide, prospective, multicentre study with the main aim of identifying genetic, clinical and environmental risk factors for diabetic complications. The FinnDiane Study has been described in detail elsewhere [14, 15]. Briefly, all adult patients with type 1 diabetes from 77 study centres were asked to participate, and the participation rate was $78 \%$. Type 1 diabetes was defined by age at onset of diabetes $<40$ years, C-peptide $\leq 0.3 \mathrm{nmol} / 1$ or insulin treatment initiation within 1 year of diagnosis if C-peptide was not measured. Written informed consent was obtained from each patient. The local ethics committees have approved the study protocol and the study has been carried out in accordance with the Declaration of Helsinki. The data include $10 \%$ of the adult patients with type 1 diabetes in Finland and the geographical distribution of the patients is similar to the distribution of the Finnish background population.

At baseline, patients underwent a thorough clinical investigation that took place in conjunction with a regular visit to the attending physician. Details of clinical characteristics of patients, including age at diagnosis, insulin therapy and diabetic complications, were obtained from medical records by the physician using a standardised questionnaire. At the baseline visit the measurement of height, weight and blood pressure was performed. In addition, fasting blood samples were drawn for the measurement of $\mathrm{HbA}_{1 \mathrm{c}}$, lipids and creatinine. The glomerular filtration rate (eGFR) was estimated using the MDRD-4 (Modification of diet in renal disease) equation [16, 17].

Data on macrovascular disease (MVD) and progression to end-stage renal disease (ESRD) were retrieved from follow-up visits, medical files or the Causes of Death Register for all patients until 31 December 2008. MVD was defined as the presence of coronary heart disease, acute myocardial infarction, stroke, coronary revascularisation or 
amputation. All amputations were pooled regardless of the aetiology. ESRD was defined as dialysis or kidney transplantation. Patients were divided into four groups based on their complication status: neither MVD nor ESRD, MVD only, ESRD only or both MVD and ESRD.

The FinnDiane data were linked to the Drug Prescription Register (DPR) in order to obtain information on all purchases of outpatient prescription medications. The Social Insurance Institution of Finland has maintained the DPR since 1994. The DPR contains the costs of prescribed, purchased and reimbursed medications in outpatient care. The data obtained from this register included the patient's unique identification number, total cost (retail price, which consists of the reasonable wholesale price, coverage for pharmacies and value added tax) of the purchase and Anatomic Therapeutic Chemical (ATC) classification code of the product, based on the 2009 ATC Index version. Over-the-counter medications and medications dispensed during a hospital stay are not recorded in the DPR.

Based on data from the Social Insurance Institution [18] the total sales of medications were $€ 2.7$ billion in Finland in 2008. Of the total sales, $73 \%$ were prescription medications used in outpatient care, $11 \%$ were over-the-counter medications and $16 \%$ were used in inpatient care. The distribution of these costs has remained fairly stable during the previous years [18].

The ATC group A10 refers to diabetes medications (insulins and analogues, oral hypoglycaemic drugs); medications related to comorbidity were all other than A10. Medications related to comorbidity were further divided into cardiovascular medication (C), immunosuppressants (L04), peritoneal dialytics (B05D), erythropoietin (B03XA) and other remaining medications. Annual and 11-year cumulative outpatient costs of prescription medication for each patient were calculated for the period between 1998 and 2008. All patients who were diagnosed with type 1 diabetes before 1998 were included in the study. All costs were adjusted for inflation to 2008 euro levels by using the Consumer Price Index (Statistics Finland).

In order to find out whether duration of diabetes has an effect on the cumulative costs, the patient population was divided into 10-year duration of diabetes groups in 1998; $(0-9,10-19,20-29,30-39$ and $\geq 40$ years $)$, and cumulative costs were evaluated according to these groups.

Statistical analysis The data are expressed as means \pm SD for normally distributed variables, as medians with interquartile range for non-normally distributed values and as percentages. Differences between groups for normally distributed variables were tested by using ANOVA and non-parametric data with Kruskal-Wallis tests. Frequencies were tested with Pearson's $\chi^{2}$ test. Because some patients had incurred considerably high costs, the 11-year cumula- tive cost data of medications were asymmetrically skewed to the right, which is a typical distribution for healthcare cost data. Therefore, generalised linear mixed models (GLMMs) under gamma distribution and log link were used to evaluate the 11-year cumulative costs and covariates associated with these costs [19]. A model built on the GLMM framework allows fitting of the data under nonnormal distribution assumptions, yielding a more flexible modelling strategy. Gamma distribution was chosen in order to remove heteroscedasticity in the error variances and to achieve precise estimates [20]. All models were adjusted for age, sex, BMI and contributing years. Contributing years consisted of the patients' follow-up years between 1998 and 2008. In addition, all variables with a $p$ value of $<0.05$ were included in the final multivariate model (duration of diabetes, total insulin dose/day as well as complication status and contributing years of ESRD and MVD). In the presence of diabetic complications $\mathrm{HbA}_{1 \mathrm{c}}$, smoking status, waist circumference, lipids and blood pressure were not significantly associated with costs and were excluded from the final models. The most likely reason for this is that those variables are strongly associated with diabetic complications per se and thus, in the presence of complications, the independent effects of these variables are not significant. Statistical analyses were performed using SAS 9.2 version (SAS Institute Inc, Cary, NC, USA). A $p$ value of $<0.05$ was considered statistically significant.

\section{Results}

This study comprised 3,717 patients with type 1 diabetes, of whom $51 \%$ were men. Mean age of the patients was $39.1 \pm 11.4$ years and mean duration of diabetes $30.9 \pm$ 11.4 years. Almost a quarter of the patients $(n=880)$ had MVD and/or ESRD. The characteristics of the patients with respect to complication status were as could be expected (Table 1). Patients with complications had higher blood pressure, worse dyslipidaemia and poorer glycaemic control. On the other hand, BMI values were lower, probably due to renal cachexia. No differences were observed regarding smoking. A total of $370(10 \%)$ patients died during the follow-up period from 1998 to 2008 .

Complication status and cumulative outpatient cost of prescription medication The observed 11-year outpatient medication costs were $€ 11,000$ per patient in those without complications, $€ 15,200$ in those with MVD only, $€ 80,900$ in those with ESRD only, and $€ 67,200$ if both MVD and ESRD were present. The average annual costs were $€ 1,000$, $€ 1,600, € 8,000$ and $€ 7,500$, respectively. On average, men's costs were $16 \%$ higher than women's. The adjusted 
Table 1 Baseline clinical characteristics of patients according to complication status $(N=3,717)$

\begin{tabular}{|c|c|c|c|c|}
\hline Characteristic & No $\mathrm{MVD}^{\mathrm{a}}$ or $\mathrm{ESRD}^{\mathrm{b}}$ & MVD only & ESRD only & Both MVD and ESRD \\
\hline$n$ & 2,837 & 385 & 221 & 274 \\
\hline Men $(\%)$ & 48 & $55^{*}$ & 55 & $66^{* * *}$ \\
\hline Age (years) & $36.9 \pm 11.4$ & $50.1 \pm 10.5^{* * *}$ & $39.9 \pm 8.6 * * *$ & $46.4 \pm 8.4 * * *$ \\
\hline Age at diabetes onset (years) & $14.0(9.0-23.0)$ & $15.0(10.0-23.0)$ & $10.0(7.0-14.0)^{* * *}$ & $13.0(8.0-17.0)^{* * *}$ \\
\hline Systolic blood pressure (mmHg) & $131 \pm 16$ & $143 \pm 20 * * *$ & $149 \pm 22 * * *$ & $152 \pm 25^{* * *}$ \\
\hline Diastolic blood pressure (mmHg) & $79 \pm 9$ & $79 \pm 10$ & $86 \pm 11 * * *$ & $83 \pm 12 * * *$ \\
\hline Body mass index $\left(\mathrm{kg} / \mathrm{m}^{2}\right)$ & $25.1 \pm 3.4$ & $25.9 \pm 3.7 * * *$ & $24.6 \pm 3.8$ & $24.6 \pm 4.1^{*}$ \\
\hline Waist circumference $(\mathrm{cm})$ & $85.0 \pm 10.7$ & $89.2 \pm 12.5^{* * *}$ & $87.4 \pm 12.4^{* *}$ & $90.6 \pm 12.6^{* * *}$ \\
\hline $\mathrm{HbA}_{1 \mathrm{c}}(\%)$ & $8.4 \pm 1.4$ & $8.6 \pm 1.3 * *$ & $8.9 \pm 1.9 * * *$ & $9.0 \pm 1.6^{* * *}$ \\
\hline Total cholesterol (mmol/l) & $4.9 \pm 1.0$ & $5.1 \pm 0.9 * *$ & $5.6 \pm 1.2 * * *$ & $5.4 \pm 1.3 * * *$ \\
\hline Triacylglycerol (mmol/l) & $0.97(0.74-1.35)$ & $1.13(0.85-1.53)^{* * *}$ & $1.58(1.14-2.30)^{* * *}$ & $1.60(1.15-2.36)^{* * *}$ \\
\hline HDL-cholesterol (mmol/l) & $1.36 \pm 0.38$ & $1.28 \pm 0.37 * * *$ & $1.16 \pm 0.40 * * *$ & $1.14 \pm 0.41 * * *$ \\
\hline LDL-cholesterol (mmol/1) & $2.93(2.44-3.52)$ & $3.11(2.57-3.61)^{* * *}$ & $3.50(2.80-4.20)^{* * *}$ & $3.36(2.77-3.92)^{* * *}$ \\
\hline $\mathrm{eGFR}^{\mathrm{c}}\left(\mathrm{ml} \mathrm{min} \min ^{-1}\left[1.73 \mathrm{~m}^{2}\right]^{-1}\right)$ & $84(71-98)$ & $68(54-83)^{* * *}$ & $12(10-30)^{* * *}$ & $10(8-18)^{* * *}$ \\
\hline Insulin dose $(U) /$ day & $51.0(40.0-64.0)$ & $44.0(35.0-58.5)^{* * *}$ & $47.0(38.0-60.0)^{*}$ & $47.0(36.0-60.0)^{* * *}$ \\
\hline Current smoking (\%) & 23 & 19 & 24 & 22 \\
\hline Died during 1998-2008, $n(\%)$ & $88(3.1)$ & $81(21.0)^{* * *}$ & $55(24.9)^{* * *}$ & $146(53.3)^{* * *}$ \\
\hline Duration of diabetes in 1998 (years), $n(\%)$ & & $* * *$ & $* * *$ & $* * *$ \\
\hline $0-9$ & $769(27.1)$ & $9(2.3)$ & $4(1.8)$ & $3(1.1)$ \\
\hline $10-19$ & $899(31.7)$ & $44(11.4)$ & $37(16.7)$ & $28(10.2)$ \\
\hline $20-29$ & $743(26.2)$ & $116(30.1)$ & $114(51.6)$ & $79(28.8)$ \\
\hline $30-39$ & 329 (11.6) & $150(39.0)$ & $61(27.6)$ & $124(45.3)$ \\
\hline$\geq 40$ & $97(3.4)$ & $66(17.2)$ & $5(2.3)$ & $40(14.6)$ \\
\hline
\end{tabular}

Data are means $\pm \mathrm{SD}$, median (interquartile range) or $\%$

${ }^{a}$ MVD denotes coronary heart disease, myocardial infarction, stroke, coronary revascularisation or amputation

${ }^{\mathrm{b}}$ ESRD denotes dialysis or kidney transplantation

${ }^{c}$ eGFR $=$ estimated glomerular filtration rate; kidney transplantation patients eGFR $=10$

$*_{p}<0.05, * * p<0.01, * * * p<0.001$ (reference group no MVD or ESRD)

cumulative cost of medications increased 56\% when MVD was present (Fig. 1) compared with the cost for patients without any complications. Cost increased significantly when ESRD was present, being four times higher. With regard to the medications related to comorbidity, the cost was 15 times higher in the patients with ESRD. The cumulative cost of diabetes medications remained quite stable irrespective of complication status, varying between $€ 5,900$ and $€ 8,200$, whereas the medication costs related to comorbidity increased dramatically according to the complication status groups from $€ 3,800$ in patients without complications to $€ 60,500$ in patients with both MVD and ESRD.

Diabetes medications accounted for $68 \%$ of the total cost for patients without complications whereas the corresponding proportion was $40 \%$ for patients with MVD only and $8 \%$ for patients with ESRD or both MVD and ESRD. Cardiovascular medications accounted for $12 \%$ of the total cost in the group without complications, $30 \%$ in the group with MVD only, 7\% in the group with ESRD only and 9\% in the group with both MVD and ESRD. Immunosuppressants accounted for $38 \%$ of the costs if ESRD was present and 33\% if both MVD and ESRD were present. Peritoneal dialytics generated the second highest costs in these two groups, $28 \%$ and $27 \%$, respectively. The proportion of erythropoietin costs was between $1 \%$ and $3 \%$ without ESRD and $10 \%$ with ESRD. The major contributors to the costs in patients with type 1 diabetes were immunosuppressants, which are predominantly used after kidney transplantation, and peritoneal dialytics, followed by erythropoietin. These medications together accounted for $70 \%$ and $76 \%$ of the costs in patients with both ESRD and MVD or with only ESRD, respectively.

Duration of diabetes and cumulative outpatient cost of prescription medication The adjusted total cost for medication without complications seemed to be quite stable 


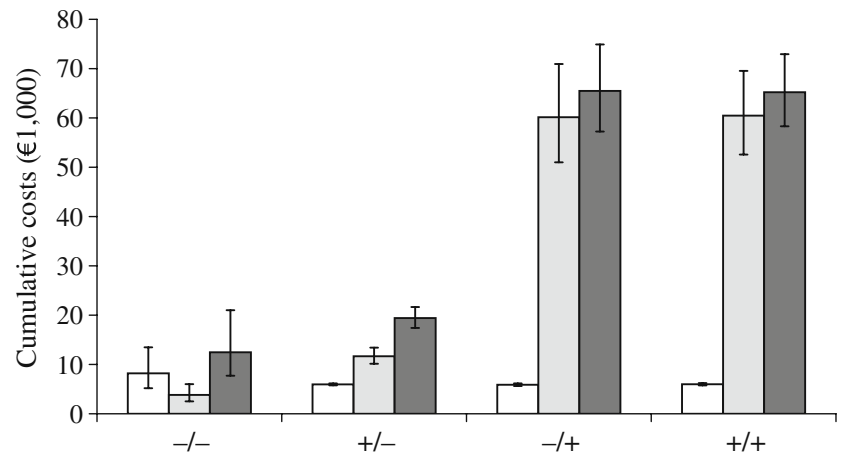

Fig. 1 The 11-year cumulative cost $(95 \% \mathrm{CI})$ of diabetes medication (white bars), medication related to comorbidity (light grey bars), and all medications (dark grey bars) in outpatients with type 1 diabetes according to complication status. -/- denotes no MVD or ESRD, +/denotes MVD only, $-/+$ denotes ESRD only and $+/+$ denotes both MVD and ESRD. All costs were adjusted for age, sex, BMI, complication status and contributing years of follow-up time. In addition diabetes medications were adjusted for total insulin dose/day, medications related to comorbidity were adjusted for duration of diabetes as well as contributing years of ESRD, and all medications were adjusted for total insulin dose/day and contributing years of ESRD

regardless of duration of diabetes (Table 2). Only those who had the shortest duration ( $0-9$ years) had slightly lower costs compared with those with the longest duration ( $\geq 40$ years) $(€ 11,600$ vs $€ 14,000 p=0.03)$. If MVD was present the costs varied between $€ 16,000$ and $€ 20,100$. Meanwhile, when ESRD or both MVD and ESRD were present the adjusted costs varied between $€ 67,400$ and $€ 75,600$. However, the differences were not significant among the duration groups.

As seen in Table 2, those who had no complications and the shortest duration of diabetes (0-9 years) had a higher cost of diabetes medication $(p=0.01)$ than those who had had diabetes for 40 years or more. On the other hand, their cost of medication related to comorbidity was slightly lower $(p=0.05)$ than the subgroup of patients with the longest duration of diabetes ( $\geq 40$ years). Similarly, those with MVD and with shorter duration of diabetes (10-19 and 20-29 years) had a significantly higher cost of diabetes medication ( $p=0.0001$ and $p=0.03)$ than those with the longest duration ( $\geq 40$ years). Notably, when ESRD was present the cumulative cost of diabetes medication was considerably lower than for those without complications in all 10-year duration groups.

Without complications the cost of medications related to comorbidity were fairly low in all 10-year duration groups (€2,500-€4,700) and no differences were observed among the groups. In contrast, these costs more than doubled in those with MVD and increased considerably further in those with ESRD in all 10-year groups. However, no differences were observed among the duration groups.

\section{Discussion}

Our results show that the presence of severe diabetic complications increases the cumulative cost of outpatient prescription medication considerably in patients with type 1 diabetes. Notably, the presence of ESRD greatly increases the cost compared with the presence of MVD without ESRD. Our data also clearly show that the 11-year cumulative cost of diabetes medication remained rather stable irrespective of complication status and duration of diabetes. The cost of medication related to comorbidity was low in patients without severe complications regardless of the duration of diabetes. However, in all duration groups the costs increased substantially when ESRD was present. Since no considerable differences were observed in the cost of diabetes medications between complication groups or duration of diabetes groups, the increase was entirely due to the cost of medications related to comorbidity, especially ESRD.

It is well established that the treatment of ESRD considerably increases the total cost of diabetes $[6,21]$. In the US in 2006, the mean annual cost of peritoneal dialysis was $\$ 53,000$ per patient and haemodialysis was $\$ 72,000$ per patient [22]. In a German study [23] the mean total dialysis-related costs were $€ 55,000$ per patient in 2006 , with $55 \%$ accounting for dialysis procedures and $22 \%$ dialysis-related medications. Our study also showed that medications associated with renal failure incur the highest costs of outpatient prescription medications in type 1 diabetes. It has been estimated that the prevalence of ESRD among patients with type 1 diabetes is approximately $1-$ $2.5 \%$ [21]. Similarly in Finland, patients with ESRD represent only $1.5 \%$ of all patients with type 1 diabetes (Finnish Kidney Register). However, extrapolation of our results to all patients with type 1 diabetes showed that they incur more than $10 \%$ of all outpatient prescription medication costs. Although the incidence of ESRD has decreased $[24,25]$, the number of ESRD patients is still increasing in Finland as well as worldwide due to the increasing incidence of diabetes, especially type 2 diabetes [26]. From this point of view, our findings together with previous studies highlight the importance of prevention, early diagnosis and intensive treatment of chronic kidney disease. Therefore, all efforts that could prevent or slow down the progression of diabetic nephropathy to ESRD may also reduce the total cost of outpatient medications for type 1 diabetes.

When ESRD was present the cost of diabetes medication was substantially lower in all 10-year duration groups compared with patients without complications. There are some possible reasons for this observation. First of all, diabetes patients with decreasing GFR require reduced quantities of insulin [27]. When GFR drops to between 10 
Table 2 The 11-year cumulative cost of diabetes medication, medication related to comorbidity, and all medications according to the duration of diabetes and complication status in outpatients with type 1 diabetes

\begin{tabular}{|c|c|c|c|}
\hline \multirow[t]{2}{*}{ Duration of diabetes in 1998 (years) } & \multicolumn{3}{|l|}{ Cumulative costs $(€)$} \\
\hline & Diabetes medications $^{\mathrm{a}}$ & Medications related to comorbidity ${ }^{\mathrm{b}}$ & All medications ${ }^{\mathrm{c}}$ \\
\hline \multicolumn{4}{|l|}{$0-9(n=785)$} \\
\hline No MVD/ESRD & $8,580(8,420-8,750)^{* *}$ & $2,470(2,080-2,920)^{*}$ & $11,580(10,970-12,230)^{*}$ \\
\hline \multicolumn{4}{|l|}{$10-19(n=1,008)$} \\
\hline No MVD/ESRD & $8,170(8,040-8,310)$ & $3,660(3,170-4,240)$ & $12,230(11,670-12,820)$ \\
\hline MVD & $7,610(7,050-8,230)^{* * *}$ & $11,610(8,430-15,990)$ & $20,080(16,340-24,680)$ \\
\hline ESRD or MVD/ESRD & $5,050(4,600-5,540)$ & $68,770(56,290-84,010)$ & $75,580(62,660-91,160)$ \\
\hline \multicolumn{4}{|l|}{$20-29(n=1,052)$} \\
\hline No MVD/ESRD & $8,010(7,870-8,160)$ & $4,560(3,880-5,360)$ & $12,970(12,310-13,660)$ \\
\hline MVD & $6,740(6,440-7,050)^{*}$ & $10,920(9,010-13,240)$ & $17,940(15,850-20,300)$ \\
\hline ESRD or MVD/ESRD & $5,520(5,230-5,810)$ & $67,440(60,370-75,350)$ & $73,780(66,500-81,860)$ \\
\hline \multicolumn{4}{|l|}{$30-39(n=664)$} \\
\hline No MVD/ESRD & $7,890(7,660-8,120)$ & $4,740(3,660-6,130)$ & $13,770(12,670-14,960)$ \\
\hline MVD & $6,470(6,220-6,730)$ & $10,780(9,160-12,690)$ & $17,420(15,690-19,350)$ \\
\hline ESRD or MVD/ESRD & $5,230(4,950-5,530)$ & $67,910(60,520-76,200)$ & $73,150(65,710-81,430)$ \\
\hline \multicolumn{4}{|l|}{$\geq 40(n=208)$} \\
\hline No MVD/ESRD & $7,930(7,520-8,370)$ & $4,180(2,610-6,700)$ & $13,970(12,010-16,250)$ \\
\hline MVD & $6,150(5,770-6,560)$ & $9,750(7,470-12,720)$ & $15,970(13,460-18,960)$ \\
\hline ESRD or MVD/ESRD & $5,820(5,190-6,530)$ & $63,060(49,680-80,050)$ & $67,430(54,020-84,170)$ \\
\hline
\end{tabular}

Data are presented as least square means $(95 \% \mathrm{CI})$

All costs were adjusted for age, sex, BMI, duration of diabetes and contributing years of follow-up time

${ }^{a}$ In addition, adjusted for total insulin dose/day and complication status as applicable

${ }^{\mathrm{b}}$ In addition, adjusted for complication status as well as contributing years of MVD and ESRD as applicable

${ }^{\mathrm{c}}$ In addition, adjusted for total insulin dose/day as well as contributing years of MVD and ESRD as applicable

${ }^{*} p<0.05, * * p<0.01, * * * p<0.001$ (reference group $\geq 40$ years duration of diabetes)

$p$ values represent comparisons between duration groups inside complication status groups

and $50 \mathrm{ml} / \mathrm{min}$, representing pre-dialysis status, a $25 \%$ reduction in the total insulin dose is recommended and, once the GFR drops below $10 \mathrm{ml} / \mathrm{min}$ (as is the case in ESRD), the recommended reduction is 50\% [28]. In addition, changes in dietary intake and exercise may decrease insulin requirements. Finally, the data from the DPR included only prescribed medications used in outpatient care. Thus, diabetes medications used in hospital were not included. The same trend was seen among patients with MVD only. At the baseline visit they had approximately $20 \%$ lower eGFR and 15\% lower insulin dose/day than those without complications. Although patients were not diagnosed with ESRD, undoubtedly a subset of them had reduced kidney function.

There are a number of studies on medication costs in diabetes. However, longitudinal studies on medication costs in type 1 diabetes are rare, especially in Finland, the country with the world's highest incidence of type 1 diabetes. To our knowledge this is one of the first studies that has estimated longitudinal medication costs by linking detailed clinical data from a nationally representative cohort of patients with type 1 diabetes to the drug register data. Mostly, medication studies have been based only on national registers without precise knowledge of the type of diabetes and with cross-sectional study design. Thus, a comparison of our findings with previous studies is difficult. It has to be emphasised that the study population in the present study is undoubtedly well characterised regarding the type of diabetes, clinical characteristics, medical history and the presence of diabetic complications. In consequence, we were able to take into account patients' BMI and insulin dose when analysing the cost of diabetes medication, which made our results more detailed and reliable. Moreover, our study population was fairly representative, including $10 \%$ of all adult patients with type 1 diabetes in Finland. Also the geographical distribution of the patients was similar to the general distribution of people in Finland.

Previous studies $[8,10]$ have compared prescription medication costs in outpatients with type 1 diabetes and 
non-diabetic controls. According to these studies annual medication costs were 8 to 12 times higher in the patients with diabetes than in the non-diabetic individuals. Our study, instead, compared the prescription medication costs among patients with type 1 diabetes according to the presence of complications and duration of diabetes. We found that there is a large variation among patients with type 1 diabetes.

The present study, however, has some limitations that need to be considered. First, the main focus of this longitudinal study was to analyse the costs of outpatient prescription medications in patients with type 1 diabetes. Medications dispensed during hospital stays and over-thecounter medications, which are not prescribed by the physician, are not recorded in the DPR. The cost of beddays in hospital includes medications costs. Therefore, the accurate patient-level data of inpatient costs of medication would have been difficult to calculate, especially during the long follow-up time. Nevertheless, the proportion of outpatient prescription medications represents approximately three-quarters of total medication sales, whereas the proportion of the medications used in hospitals accounts for only $16 \%$ [18]. Obviously, the more complications patients have, the more hospitalisation periods they may have. According to a previous Finnish study [29] the total number of patients with type 1 diabetes hospitalised due to complications almost doubled when duration of diabetes increased from 9.5 to 16.5 years. Therefore, it is probable that among patients with type 1 diabetes and severe complications the outpatient costs underestimate the true medication cost. Moreover, with regard to dialysis methods, peritoneal dialytics are dispensed through pharmacies, but haemodialysis fluids are mainly administered by hospitals. Thus, haemodialysis fluids are not registered in the DPR. Patients staying permanently in institutional care are not entitled to drug reimbursement, and the drugs dispensed for them are not registered in the DPR either.

Second, although the coverage and accuracy of the Finnish DPR is very high, only the data on reimbursed medications are available from this register. The costs of medications are reimbursed after the holder of the marketing authorisation has applied for reimbursement and demonstrated a reasonable wholesale price, which has been confirmed by the Pharmaceuticals Pricing Board [30]. Moreover, the Pharmaceuticals Pricing Board can restrict the basis of reimbursement for a medicinal product to a precisely defined diagnosis. For example, medications for erectile dysfunction caused by diabetes are not justified for reimbursement, and therefore they do not appear in the register. Some changes due to legislative amendments have also affected the content and coverage of this register. Since 2006 the coverage of the DPR has improved. The fixed deductible rate per purchase for medications was given up and, instead, a certain percentage of the price was calculated on the basis of reimbursement category. As a consequence of this, inexpensive medications that had not reached the limit before are now recorded in the database. Moreover, since 2007 medications funded by employers have also been recorded in the register. Nevertheless, the DPR database provides an excellent resource to study medication utilisation and costs. Furthermore, since all patients in this study have been affected by the above mentioned changes in the same way, we are confident that these changes have had only a minor effect on the results.

Finally, we did not receive comprehensive information on comorbidities unrelated to diabetes or its complications, such as other autoimmune diseases or cancer. Thus, we were not able to control for these unrelated comorbidities in the multivariate model, which might have had some minor effects on the results.

In conclusion, although diabetes itself generates high medication costs, our findings highlight the high cost of renal failure; it dramatically increases the total cost of prescription medications in outpatients with type 1 diabetes. On the contrary, the cost of diabetes medication is stable irrespective of complication status and duration of diabetes. Although the incidence of ESRD has decreased, the number of patients with ESRD will continue to rise worldwide due to the growing number of individuals with diabetes. Therefore, both healthcare professionals and policymakers should be aware that all interventions to prevent or delay ESRD will save not only medication costs, but also the long-term costs of diabetes as a whole. In preventing complications, healthcare professionals should pay particular attention to motivating individuals with diabetes to conduct better self-care from the very beginning of the disease process.

Acknowledgements The authors would like to acknowledge all of the physicians and nurses at each of the FinnDiane centres participating in patient recruitment and characterisation. This research was supported by grants from the Folkhälsan Research Foundation, Wilhelm and Else Stockmann Foundation and Liv och Hälsa Foundation.

Duality of interest The authors declare that there is no duality of interest associated with this manuscript.

\section{References}

1. Dahlquist G, Bennich SS, Kallen B (1996) Intrauterine growth pattern and risk of childhood onset insulin dependent (type I) diabetes: population based case-control study. BMJ 313:11741177

2. DIAMOND Project Group (2006) Incidence and trends of childhood type 1 diabetes worldwide 1990-1999. Diabet Med 23:857-866 
3. Harjutsalo V, Sjoberg L, Tuomilehto J (2008) Time trends in the incidence of type 1 diabetes in Finnish children: a cohort study. Lancet 371:1777-1782

4. Patterson CC, Dahlquist GG, Gyurus E, Green A, Soltesz G, EURODIAB Study Group (2009) Incidence trends for childhood type 1 diabetes in Europe during 1989-2003 and predicted new cases 2005-20: a multicentre prospective registration study. Lancet 37:2027-2033

5. Andersen AR, Christiansen JS, Andersen JK, Kreiner S, Deckert T (1983) Diabetic nephropathy in type 1 (insulin-dependent) diabetes: an epidemiological study. Diabetologia 25:496-501

6. American Diabetes Association (2008) Economic costs of diabetes in the U.S. in 2007. Diabetes Care 31:596-615

7. Daneman D (2006) Type 1 diabetes. Lancet 367:847-858

8. Bruno G, Karaghiosoff L, Merletti F et al (2008) The impact of diabetes on prescription drug costs: the population-based Turin study. Diabetologia 51:795-801

9. Rathmann W, Haastert B, Icks A, Giani G (2007) Trends in outpatient prescription drug costs in diabetic patients in Germany, 1994-2004. Diabetes Care 30:848-853

10. Reunanen A, Kangas T, Martikainen J, Klaukka T (2000) Nationwide survey of comorbidity, use, and costs of all medications in Finnish diabetic individuals. Diabetes Care 23:1265-1271

11. Evans JM, MacDonald TM, Leese GP, Ruta DA, Morris AD (2000) Impact of type 1 and type 2 diabetes on patterns and costs of drug prescribing: a population-based study. Diabetes Care 23:770-774

12. Kangas T (2002) The consumption and direct costs of healthcare services among persons with diabetes in Helsinki. A case controlled cross-sectional study of fiscal year 1997. The Social Insurance Institution, Finland, Studies in social security and health 67, Gummerus Kirjapaino Oy, Jyväskylä [in Finnish]

13. Johnson JA, Pohar SL, Majumdar SR (2006) Healthcare use and costs in the decade after identification of type 1 and type 2 diabetes: a population-based study. Diabetes Care 29:2403-2408

14. Thorn LM, Forsblom C, Fagerudd J et al (2005) Metabolic syndrome in type 1 diabetes: association with diabetic nephropathy and glycemic control (the FinnDiane study). Diabetes Care 28:2019-2024

15. Saraheimo M, Teppo AM, Forsblom C, Fagerudd J, Groop PH (2003) Diabetic nephropathy is associated with low-grade inflammation in type 1 diabetic patients. Diabetologia 46:1402-1407

16. Levey AS, Bosch JP, Lewis JB, Greene T, Rogers N, Roth D (1999) A more accurate method to estimate glomerular filtration rate from serum creatinine: a new prediction equation. Modifica- tion of diet in renal disease study group. Ann Intern Med 130:461-470

17. Levey AS, Coresh J, Greene T et al (2006) Chronic kidney disease epidemiology collaboration. Using standardized serum creatinine values in the modification of diet in renal disease study equation for estimating glomerular filtration rate. Ann Intern Med $145: 247-254$

18. National Agency for Medicines and Social Insurance Institution (2009) Finnish statistics on medicines 2008. Edita Prima Oy, Helsinki

19. Littell RC (ed) (2006) SAS for mixed models, 2nd edn. SAS Institute Inc., Cary, NC

20. Manning WG, Mullahy J (2001) Estimating log models: to transform or not to transform? J Health Econ 20:461-494

21. Gordois A, Scuffham P, Shearer A, Oglesby A (2004) The healthcare costs of diabetic nephropathy in the United States and the United Kingdom. J Diabetes Complications 18:18-26

22. Foley RN, Collins AJ (2009) The growing economic burden of diabetic kidney disease. Curr Diab Rep 6:460-465

23. Icks A, Haastert B, Gandjour A et al (2010) Costs of dialysis-a regional population-based analysis. Nephrol Dial Transplant $25: 1647-1652$

24. Burrows NR, Li Y, Geiss LS (2010) Incidence of treatment for end-stage renal disease among individuals with diabetes in the U.S. continues to decline. Diabetes Care 33:73-77

25. Finne P, Reunanen A, Stenman S, Groop PH, Gronhagen-Riska C (2005) Incidence of end-stage renal disease in patients with type 1 diabetes. JAMA 294:1782-1787

26. Wild S, Roglic G, Green A, Sicree R, King H (2004) Global prevalence of diabetes: estimates for the year 2000 and projections for 2030. Diabetes Care 27:1047-1053

27. Biesenbach G, Raml A, Schmekal B, Eichbauer-Sturm G (2003) Decreased insulin requirement in relation to GFR in nephropathic type 1 and insulin-treated type 2 diabetic patients. Diabet Med 20:642-645

28. Shrishrimal K, Hart P, Michota F (2009) Managing diabetes in hemodialysis patients: Observations and recommendations. Cleve Clin J Med 76:649-655

29. Akkanen MJ (2007) Inpatient hospital care and its costs among type 1 diabetic patients in Finland - a nationwide longitudinal study. Department of Public Health in Helsinki. University of Helsinki, Edita Prima Oy

30. The Health Insurance Act (1224/2004). Ch. 5 and 6, as amended by Act $802 / 2008$ [unofficial translation]. The original version of the Act is available from www.stm.fi/en/ministry/boards/pharmaboard/ legislation, accessed 15 November 2010 